\title{
Simple model of adsorption on external surface of carbon nanotubes-a new analytical approach basing on molecular simulation data
}

\author{
Sylwester Furmaniak • Artur P. Terzyk • Piotr \\ A. Gauden • Peter J.F. Harris • Marek Wiśniewski • \\ Piotr Kowalczyk
}

Received: 12 February 2010 / Accepted: 31 May 2010 / Published online: 18 June 2010

(C) The Author(s) 2010. This article is published with open access at Springerlink.com

\begin{abstract}
Nitrogen adsorption on carbon nanotubes is widely studied because nitrogen adsorption isotherm measurement is a standard method applied for porosity characterization. A further reason is that carbon nanotubes are potential adsorbents for separation of nitrogen from oxygen in air. The study presented here describes the results of GCMC simulations of nitrogen (three site model) adsorption on single and multi walled closed nanotubes. The results obtained are described by a new adsorption isotherm model proposed in this study. The model can be treated as the tube analogue of the GAB isotherm taking into account the lateral adsorbateadsorbate interactions. We show that the model describes the simulated data satisfactorily. Next this new approach is applied for a description of experimental data measured on different commercially available (and characterized using HRTEM) carbon nanotubes. We show that generally a quite good fit is observed and therefore it is suggested that the observed mechanism of adsorption in the studied mate-
\end{abstract}

Electronic supplementary material The online version of this article (doi:10.1007/s10450-010-9223-4) contains supplementary material, which is available to authorized users.

S. Furmaniak · A.P. Terzyk $(\bowtie) \cdot$ P.A. Gauden · M. Wiśniewski Physicochemistry of Carbon Materials Research Group, Department of Chemistry, N. Copernicus University,

Gagarin St. 7, 87-100 Toruń, Poland

e-mail: aterzyk@chem.uni.torun.pl

url: http://www.chem.uni.torun.pl/ aterzyk/

P.J.F. Harris

Centre for Advanced Microscopy, University of Reading,

Whiteknights, Reading RG6 6AF, UK

P. Kowalczyk

Applied Physics, RMIT University, GPO Box 2476V, Melbourn,

Victoria 3001, Australia rials is mainly determined by adsorption on tubes separated at large distances, so the tubes behave almost independently.

Keywords Adsorption - GCMC · Carbon nanotubes · Nitrogen · Surface area

\section{Introduction}

To characterize the porous structure of various adsorbents argon and/or nitrogen adsorption isotherms measured for the temperature of their respective boiling points are usually used (Bansal and Goyal 2005; Lowell et al. 2004; Marsh and Rodriguez-Reinoso 2006; Rouquerol et al. 1999). It is well known that $\mathrm{Ar}$ is preferred over $\mathrm{N}_{2}$ due to its spherical shape and zero electric moments. On the other hand, $\mathrm{N}_{2}$ (possessing quadrupole moment) is commonly used for routine gas adsorption measurements because much experimental evidence has been obtained in the past for nitrogen and therefore the comparison of results is easy $\left(\mathrm{N}_{2}\right.$ is used in most commercial surface area and porosity analyzers). A further reason is low operating cost. Therefore, precise quantification of $\mathrm{N}_{2}$ adsorption properties of carbon nanotubes, which should be valid for a wide range of experimental conditions, is essential to understand how best describe the heterogeneity of these materials (Agnihotri et al. 2004; Kowalczyk et al. 2005; Li et al. 2004) and to apply carbon nanotubes for adsorption related application, for example for adsorption and separation of nitrogen and oxygen mixture (identified with air) (Arora and Sandler 2005; Jiang and Sandler 2004; Zhang et al. 2008).

The process of $\mathrm{N}_{2}$ adsorption in carbon nanotubes has been modeled since the middle of the 1990s (Maddox and Gubbins 1995). It should be noted that more and more 
complicated models reproducing the structure of real carbon nanotubes (i.e. from ideal cylinders (Arora et al. 2004; Arora and Sandler 2005; Gauden et al. 2009; Harris 2009; Khan and Ayappa 1998; Kotsalis et al. 2004; Kowalczyk et al. 2005; Maddox and Gubbins 1995; Müller 2008; Ohba and Kaneko 2002; Terzyk et al. 2008; Zhang et al. 2008) to bundles (Alain et al. 2000; Arab et al. 2004; Furmaniak et al. 2008a, 2009a; Harris 2009; Jiang and Sandler 2003, 2004; Paredes et al. 2003; Wongkoblap et al. 2009; Yin et al. 1999) and tubes with defects (Arora and Sandler 2007a, 2007b; Furmaniak et al. 2009a; Gauden et al. 2009; Harris 2009; Terzyk et al. 2008; Zhang et al. 2008)) have been applied. On the other hand, despite the complex structure of carbon nanotubes confirmed by the analysis of experimental data (mutual position of nanotubes in the bundle, defects, an unavoidable by-products of a typical nanotube growth process i.e. impurities, such as carbon-coated catalyst particles, graphitic and amorphous carbons, and other carbon nanomaterials (Harris 2009)) the evaluation of the pore-size distribution of carbon nanotube sizes from the static adsorption measurements and computer simulations of nitrogen adsorption at $77 \mathrm{~K}$ calculated for the separated nanotubes has been treated as the standard method (Furmaniak et al. 2008a; Kowalczyk et al. 2005; Zhang et al. 2003). However, the above-mentioned simple procedure has been insufficient for some experimental data. In order to improve the description of experimental results Wongkoblap et al. (2009) assumed that the experimental overall adsorption isotherm on carbon nanotubes (bundles) can be split into the sum of individual contributions arising from different adsorption sites, i.e. from nanotubes and from the adsorption on surface of the impurities, as suggested by Agnihotri et al. (2006). Additionally, the methodology proposed by Agnihotri et al. $(2005,2006,2007)$ determines the fraction of accessible endohedral and interstitial sites in the sample.

It is well-known that the effect of the nanoscale curvature of nanotubes can lead to inapplicability of different approaches developed for "flat" surfaces. For example Ohba and Kaneko (2002) concluded about inapplicability of the BET analysis for evaluating of surface area of the internal and external surfaces of nanotubes. They considered $\mathrm{N}_{2}$ (single LJ centre model) adsorption on single-walled carbon nanotubes at $77 \mathrm{~K}$ and using GCMC simulation. Moreover, Ohba and Kaneko (2002) studied the properties of nitrogen molecules adsorbed on/in nanotubes. They showed that $\mathrm{N}_{2}$ adsorption isotherm had two steps caused by monolayer formation on the internal surface and filling of the residual space of the internal tube pore. Although all of the radial distribution functions of molecules adsorbed on the internal, external, and flat surfaces indicated a hexagonal packing structure, the radial distribution functions were different one from each other. In particular, the radial distribution function of the monolayer on the internal surface showed an additional peak because of the markedly curved surface. Using molecular simulations (GCMC and EMD), adsorption and self-diffusion of diatomic nitrogen (two centre LJ model) molecules inside a infinite single wall carbon nanotube have been studied by Arora et al. (2004) over a range of nanotube diameters $(0.86-1.57 \mathrm{~nm})$ and loadings at temperatures of 100 and $298 \mathrm{~K}$. A discrete organization of the nitrogen into adsorbed layers was observed at high loadings following a regular progression determined primarily by geometric considerations. The formation of an adsorbate core at the centre of the nanotube was found to increase the self-diffusion of nitrogen. A "wormlike" phase is detected for the adsorbed nitrogen in the $(15,0)$ carbon nanotube at high loadings and at $100 \mathrm{~K}$. Separation of a nitrogen (two centre LJ model)oxygen mixture by single wall carbon nanotubes has been studied by the same authors (Arora and Sandler 2005) using grand canonical Monte Carlo simulations at a range of nanotube diameters, temperatures, and pressures. In singlecomponent simulations, it was found that near saturation nitrogen molecules form only an annular layer close to the tube wall, while smaller-sized oxygen also occupies the region near the centre of the nanotube. In mixture adsorption, energetically favored nitrogen is preferentially adsorbed at low loadings. However, at high loadings oxygen replaces nitrogen due to the dominant entropic effects, and therefore a high selectivity towards oxygen is observed close to the saturation. Mass transport of pure nitrogen (two centre LJ model), pure oxygen, and their mixture has been studied at $100 \mathrm{~K}$ in a single wall carbon nanotube of $1.253 \mathrm{~nm}$ diameter (EMD). The concentration profiles, diffusivity profiles, and membrane fluxes were calculated, and it was demonstrated that by carefully adjusting the upstream and downstream pressures, a good kinetic selectivity can be achieved for air separation using single wall carbon nanotubes (Arora and Sandler 2006). Simulations performed using a carbon nanotube with a constriction showed that the size or entropic effect can be dominant (Arora and Sandler 2007a, 2007b). In particular, it was shown that an appropriate size constriction can result in large transport resistance to nitrogen (two centre LJ model) while letting oxygen pass through at a much higher rate, even though these gases have very similar molecular sizes and interaction energy. Transport of pure nitrogen (two centre LJ model), oxygen, and their mixture in singlewalled carbon nanotubes with a kink formed by bending is studied using molecular dynamics simulations (Zhang et al. 2008). The results show that a nanotube with a specified kink results in transport resistance to nitrogen, while allowing oxygen to pass even though the two gases have very similar molecular sizes. The permeability decreases while the selectivity increases with increasing the bending angle of single walled nanotubes (SWNT). The most important finding is that it is very convenient to obtain the required purity of the oxygen and permeability by adjusting the bending angle of SWNT. It should be noted that other mixtures were also studied ( $\mathrm{N}_{2}$ (single LJ model)- $\mathrm{H}_{2} \mathrm{O}$ ) (Kotsalis et al. 2004). 
Yin et al. (1999) studied adsorption of nitrogen (single LJ model) on square arrays of open and closed single-walled nanotubes with different diameters. They concluded that adsorption isotherms, in closed tubes with small tube separations, are of type I. In the case of open tubes, the extent of filling of the adsorption space is dominated by the tube diameter and the separation between tubes. Alain et al. (2000) studied exohedral and endohedral nitrogen (single LJ centre) adsorption on model SWNT forming a square array. Paredes et al. (2003) considered nanotubes modeled by grouping them in bundles forming a hexagonal array. They compared the simulations $\left(\mathrm{N}_{2}\right.$-two centre LJ model) with the experimental isotherms, achieving a good agreement between both results. In their opinion, interstitial adsorption seems to be very important. However, the adsorption capacity obtained experimentally does not reach the levels predicted by simulations. This discrepancy could be due, in their opinion, to insufficient purification of the nanotube samples and to a failure in the model, particularly in the assumption of an array of the tubes. Nitrogen (single LJ centre model) adsorption on two types of single-walled carbon nanotube hexagonal bundles (an infinite periodic hexagonal bundle that does not have an external surface, and a finite isolated hexagonal bundle that does have an external surface) at both subcritical and supercritical temperatures (77, 100, and $300 \mathrm{~K}$ ) was studied using Gibbs ensemble Monte Carlo simulation to understand the role of the external surface in the type of isotherm (Jiang and Sandler 2003). The results discussed by Jiang and Sandler demonstrate the important role of the external surface of a nanotube bundle in the character of the adsorption isotherm, and provide a substantial physical explanation for the difference between experimental observation and previous theoretical predictions. The results published by Arab et al. (2004) show that (i) including polarization effects in the interaction potential is required for the case of interaction with polar molecules, (ii) the sieving properties of SWNT depend strongly on the radius of the tubes and four domains of behavior regarding the most stable site can be found depending on the radius values, and (iii) the groove sites are more favorable energetically for $\mathrm{N}_{2}$. Wongkoblap et al. (2009) used homogeneous (hexagonal) nanotube bundles to investigate adsorption of $\mathrm{N}_{2}$ (1 $1 \mathrm{LJ}$ or $2 \mathrm{LJ}$ models, GCMC) and Ar. The effects of nanotube diameter and the spacing between the nearest neighbor SWNT on the adsorption of those fluids were investigated to determine differences in their adsorption mechanisms. As was expected the preferential adsorption of argon and nitrogen depends on the tube size. Jiang and Sandler considered adsorption of a nitrogen (two centre LJ model) and oxygen mixture (air) on single-walled carbon nanotube hexagonal bundles (Jiang and Sandler 2004). They concluded that adsorption selectivity strongly depends on temperature but only weakly depends on the type of the bundle and that a $\mathrm{N}_{2}-\mathrm{O}_{2}$ might be separated by competitive adsorption on the carbon nanotube bundles. It is well-known that characterization of the porosity of real adsorbents is not a simple task due to the influence of the complex structure on the adsorption process.

Therefore, some authors treated the computer generated adsorption isotherms on model nanotubes as experimental data. Next, the theoretical models and the methodology of the calculation of the porosity parameters can be verified using these computer generated results. A double Langmuir model was used to describe nitrogen adsorption isotherm obtained from the GCMC simulation (single-walled nanotubes) (Arora et al. 2004). All simulated isotherms were non-Langmuirian suggesting either "multilayer" adsorption or a restructuring of the adsorbed layer with increased loading. The analysis of parameters obtained from fitting suggested that there is significant adsorption at low pressures, as indicated by the relatively high values of $k_{1}$, which increases with decreasing tube radius. At both 100 and $298 \mathrm{~K}$, the value of $N_{1}^{\max }$ (the maximum number of molecules adsorbed per unit cell) was of the same order of magnitude as $N_{2}^{\max }$ for the $(17,0)$ and $(20,0)$ SWNTs, suggesting that both "layers" make comparable contributions to the maximum loading. However, for the $(15,0)$ and $(11,0)$ SWNTs, $N_{1}^{\max }$ and $N_{2}^{\max }$ differ by 1 order of magnitude, suggesting that the application of a dual Langmuir isotherm reflects reorganization in the adsorbed layer rather than the existence of two distinguishable adsorption layers. A dual-site Langmuir-Freundlich isotherm was used to fit the pure component and mixture adsorption isotherms by Arora and Sandler (2005). A satisfactory description was observed only at high nitrogen loading.

From the short review presented above it can be concluded that nitrogen adsorption/separation on carbon nanotubes is a very important subject in their possible practical application as well as in the field of porous structure characterization. It can be also concluded that there is a lack of a model satisfactorily describing adsorption processes on external surfaces of carbon nanotubes since the process is very complex. In our first paper of this series a simple model of adsorption in the internal space of carbon nanotubes was proposed (Furmaniak et al. 2006). The most important feature of the model was taking into account the changes in maximum adsorption capacity caused by a cylindrical shape of adsorption space, i.e. in the pores having cylindrical geometry, the surface area of the upper layer (in comparison with the bottom layer) should be diminished proportionally to the radius of a cylinder.

In this study we propose a similar model, but for adsorption on the external surface of closed carbon nanotubes. As previously, it will be assumed that the mechanism of adsorption follows the scenario of BET (Brunauer et al. 1938; Rouquerol et al. 1999) and GAB (Anderson 1946; De Boer 1953; Guggenheim 1966; Rouquerol et al. 1999) models, which are widely applied in the field of adsorption science. 


\section{Model formulation}

The major assumptions of the new model are as follows:

(i) adsorption is polymolecular,

(ii) the number of layers is finite (and equal to $N$ ),

(iii) the filling of a following layer is possible only at those places where the preceding layer has just been filled,

(iv) maximum adsorption capacity of each following layer increases due to the cylindrical geometry of a tube.

Simple geometric considerations lead to the equation defining the ratio of an upper surface area (this is of course the bottom surface of following layer) of a chosen layer to a bottom surface area of this layer (this is of course the top surface of a subsequent layer):

$\alpha_{i}=\frac{r_{\text {eff }}+i \cdot \lambda}{r_{\text {eff }}+(i-1) \cdot \lambda}=\frac{1+i \cdot \frac{\lambda}{r_{e f f}}}{1+(i-1) \cdot \frac{\lambda}{r_{e f f}}}$

where $i$ is the number of a layer, $r_{\text {eff }}$ is the effective external radius of a tube, and $\lambda$ is a thickness of a layer.

Additionally, one can define the coverage for each layer (bearing in mind, that the surface of a bottom layer is larger than the surface of a subsequent layer):

$\Theta_{i}= \begin{cases}\frac{a_{1}}{a_{m}} & i=1 \\ \frac{a_{i}}{\alpha_{i-1} a_{i-1}} & i>1\end{cases}$

where $a_{m}$ is a maximal capacity in the first layer (at the vicinity of a tube), and $a_{i}$ is the value of adsorption in the $i$ th layer.

Next we can consider the relation describing the rate of adsorption in each layer (assuming, that it is proportional to the value of relative pressure, $h=p / p_{s}$, where $p_{s}$ is the saturation pressure, and to the value of bare surface area):

$r_{i}^{a}= \begin{cases}k_{1}^{a}\left(a_{m}-a_{1}\right) h \exp \left(A_{1} \Theta_{1}\right) & i=1 \\ k_{p}^{a}\left(\alpha a_{i-1}-a_{i}\right) h \exp \left(A_{p} \Theta_{i}\right) & i>1\end{cases}$

or:

$r_{i}^{a}= \begin{cases}k_{1}^{a} a_{m}\left(1-\Theta_{1}\right) h \exp \left(A_{1} \Theta_{1}\right) & i=1 \\ k_{p}^{a} \alpha_{i-1} a_{i-1}\left(1-\Theta_{i}\right) h \exp \left(A_{p} \Theta_{i}\right) & i>1\end{cases}$

In the given above equations (i.e. (3) and (4)) the intermolecular interactions between adsorbed molecules (i.e. fluidfluid) are taken into account by an empirical exponential term.

On the other hand, the rate of desorption for each layer is proportional to the value of upper surface unoccupied by adsorbed molecules in the following layer:

$r_{i}^{d}= \begin{cases}k_{1}^{d}\left(\alpha_{1} a_{1}-a_{2}\right) & i=1 \\ k_{p}^{d}\left(\alpha_{i} a_{i}-a_{i+1}\right) & 1<i<N \\ k_{p}^{d} \alpha_{i} a_{i} & i=N\end{cases}$ or:

$r_{i}^{d}= \begin{cases}k_{1}^{d} a_{m} \alpha_{1} \Theta_{1}\left(1-\Theta_{2}\right) & i=1 \\ k_{p}^{d} \alpha_{i-1} a_{i-1} \alpha_{i} \Theta_{i}\left(1-\Theta_{i+1}\right) & 1<i<N \\ k_{p}^{d} \alpha_{i-1} a_{i-1} \alpha_{i} \Theta_{i} & i=N\end{cases}$

Since at equilibrium the rate of adsorption is equal to the rate of desorption one can compare equations (4) and (6) and derive the formula connecting the relative pressure and the coverage in each layer:

$h= \begin{cases}\frac{\alpha_{1} \Theta_{1}\left(1-\Theta_{2}\right)}{K_{1}\left(1-\Theta_{1}\right)} \cdot \exp \left(-A_{1} \Theta_{1}\right) & i=1 \\ \frac{\alpha_{i} \Theta_{i}\left(1-\Theta_{i+1}\right)}{K_{p}\left(1-\Theta_{i}\right)} \cdot \exp \left(-A_{p} \Theta_{i}\right) & 1<i<N \\ \frac{\alpha_{i} \Theta_{i}}{K_{p}\left(1-\Theta_{i}\right)} \cdot \exp \left(-A_{p} \Theta_{i}\right) & i=N\end{cases}$

where:

$K_{1}=\frac{k_{1}^{a}}{k_{1}^{d}}$

$K_{p}=\frac{k_{p}^{a}}{k_{p}^{d}}$

As one can see we assume differences between the values of interaction parameters for the first and for the following layers. For the latter layers, where adsorbate-adsorbate interactions dominate, we assume the same values of interaction parameters (similarly as in the original GAB model).

Total adsorption is a sum of the adsorptions in each layer:

$a=\sum_{i=1}^{N} a_{i}=a_{m} \cdot \Theta_{1}+\sum_{i=2}^{N} \alpha_{i-1} \cdot a_{i-1} \cdot \Theta_{i}$

Since our approach has similar assumptions as the GAB model we can call it the nanotube analogue of the GAB model. However, our approach takes into consideration the lateral adsorbate-adsorbate interactions. Due to the properties of (7) and (10) (interrelation between the values of adsorption in neighboring layers) the determination of adsorption isotherms must be performed using numerical procedures. Calculations are performed in an iterative way for each pressure value; starting from zero coverage in all layers for each following layer (using bisection method) the value of adsorption is calculated basing on the values of adsorption in neighboring layers. The iterations are performed until the value of coverage in each layer does not differ from the previous value more than $1 \cdot 10^{-8}$.

\section{GCMC simulations of adsorption on carbon nanotubes}

To check the applicability of the above-proposed model, three series of carbon nanotubes, having the length of 
Table 1 Characteristics of carbon nanotubes applied as adsorbents in simulation of nitrogen adsorption isotherms (at $77.3 \mathrm{~K}$ )

\begin{tabular}{|c|c|c|c|c|c|}
\hline Notation & Tube indexes & Notation & Tube indexes & Notation & Tube indexes \\
\hline \multicolumn{2}{|c|}{ Single walled nanotubes } & \multicolumn{2}{|c|}{ Double walled nanotubes } & & \\
\hline t_w1_012 & $(12,0)$ & t_w2_016 & $(16,0),(8,0)$ & \multicolumn{2}{|c|}{ Triple walled nanotubes } \\
\hline t_w1_024 & $(24,0)$ & t_w2_024 & $(24,0),(16,0)$ & t_w3_024 & $(24,0),(16,0),(8,0)$ \\
\hline t_w1_036 & $(36,0)$ & t_w2_036 & $(36,0),(28,0)$ & t_w3_036 & $(36,0),(28,0),(20,0)$ \\
\hline t_w1_048 & $(48,0)$ & t_w2_048 & $(48,0),(40,0)$ & t_w3_048 & $(48,0),(40,0),(32,0)$ \\
\hline t_w1_060 & $(60,0)$ & t_w2_060 & $(60,0),(52,0)$ & t_w3_060 & $(60,0),(52,0),(44,0)$ \\
\hline t_w1_072 & $(72,0)$ & t_w2_072 & $(72,0),(64,0)$ & t_w3_072 & $(72,0),(64,0),(56,0)$ \\
\hline t_w1_084 & $(84,0)$ & t_w2_084 & $(84,0),(76,0)$ & t_w3_084 & $(84,0),(76,0),(68,0)$ \\
\hline t_w1_096 & $(96,0)$ & t_w2_096 & $(96,0),(88,0)$ & t_w3_096 & $(96,0),(88,0),(80,0)$ \\
\hline t_w1_108 & $(108,0)$ & t_w2_108 & $(108,0),(100,0)$ & t_w3_108 & $(108,0),(100,0),(92,0)$ \\
\hline t_w1_120 & $(120,0)$ & t_w2_120 & $(120,0),(112,0)$ & t_w3_120 & $(120,0),(112,0),(104,0)$ \\
\hline t_w1_132 & $(132,0)$ & t_w2_132 & $(132,0),(124,0)$ & t_w3_132 & $(132,0),(124,0),(116,0)$ \\
\hline t_w1_144 & $(144,0)$ & t_w2_144 & $(144,0),(136,0)$ & t_w3_144 & $(144,0),(136,0),(128,0)$ \\
\hline t_w1_156 & $(156,0)$ & t_w2_156 & $(156,0),(148,0)$ & t_w3_156 & $(156,0),(148,0),(140,0)$ \\
\hline t_w1_168 & $(168,0)$ & t_w2_168 & $(168,0),(160,0)$ & t_w3_168 & $(168,0),(160,0),(152,0)$ \\
\hline \multirow[t]{4}{*}{ t_w1_180 } & $(180,0)$ & t_w2_180 & $(180,0),(172,0)$ & t_w3_180 & $(180,0),(172,0),(164,0)$ \\
\hline & & & & \multicolumn{2}{|c|}{ Multi walled nanotubes } \\
\hline & & & & t_w4_084 & $(84,0),(76,0),(68,0),(60,0)$ \\
\hline & & & & t_w5_084 & $(84,0),(76,0),(68,0),(60,0),(52,0)$ \\
\hline
\end{tabular}

$4.23 \mathrm{~nm}$, were generated, namely: single, double and triple walled, respectively (the distance between layers was assumed as similar to the interlayer spacing in graphite). To check the influence of the number of tube layers on obtained results for one of studied nanotubes we generated the tube containing four and five walls. Table 1 shows the characteristics of all tubes.

Nitrogen adsorption isotherms $(77.3 \mathrm{~K})$ were simulated using the classical GCMC method. The cubicoid simulation boxes with a tube placed in a box centre (along $z$ axis) having dimensions $40 \times 40 \times 4.23 \mathrm{~nm}$ were applied. Periodic boundary conditions were used in all three directions, and the internal space of a tube was assumed as inaccessible for adsorbed molecules. For each adsorption point $25 \cdot 10^{6}$ iterations were performed during the equilibration, and next $25 \cdot 10^{6}$ equilibrium ones, applied for the calculation of the averages (one iteration $=$ an attempt to change the state of the system by creation, annihilation, rotation or displacement). The probability of attempts of changing a system state by creation, annihilation, and rotation and displacement (the latter one is connected with the change in angular orientation) were equal to: $1 / 3,1 / 3,1 / 6$ and $1 / 6$.

Simulation studies of nitrogen adsorption in carbon nanotubes can be performed assuming a spherical model (Alain et al. 2000; Kotsalis et al. 2004; Kowalczyk et al. 2005; Maddox and Gubbins 1995; Ohba and Kaneko 2002; Ohba et al. 2007; Wongkoblap et al. 2009; Yin et al. 1999) but if nitrogen molecules are confined in nanotubes the assumed shape of molecules is important and nitrogen should be modeled as a diatomic molecule (Arab et al. 2004; Arora et al. 2004; Arora and Sandler 2005, 2007a, 2007b; Jiang and Sandler 2003; Khan and Ayappa 1998; Müller 2008; Paredes et al. 2003; Wongkoblap et al. 2009; Zhang et al. 2008). To test the importance of molecular shape, both potential models (spherical and diatomic) were used by Wongkoblap et al. (2009) in computer simulation. It was noted that a single-centre model (assuming nitrogen to be pseudo sphere) gives greater density than the double-centre model, and secondly as the tube size is smaller the difference between the both models becomes significant. The differences between results obtained using these two models are also reported in the literature for nitrogen adsorption on carbon blacks (Bottani and Bakaev 1994; Do and Do 2005). The results published by Do et al. show that for twocentre model simulation the results agree quite well with experimental data, but the single-site model shows an overprediction of the completion of monolayer and higher layers. Additionally, simulated isosteric heat of the two-centre LJ model (for adsorption on carbon bundles) is greater than that for a single-site model in the sub-monolayer region (the opposite is true when it reaches a monolayer). This is because a non-spherical molecule lies flat on the graphene wall and this leads to the stronger fluid-solid interaction, but this effect does not occur in the multilayer region (Bottani and Bakaev 1994). Similar results were reported by Müller (2008) and Khan and Ayappa (1998) from the GCMC stud- 
Table 2 The values of parameters applied in simulations

${ }^{\mathrm{a}}$ Centre of mass.

\begin{tabular}{lllllll}
\hline Molecule & Centre & $\sigma[\mathrm{nm}]$ & $\varepsilon / k_{B}[\mathrm{~K}]$ & $q / e$ & Bond length & References \\
\hline $\mathrm{N}_{2}$ & $\mathrm{~N}$ & 0.331 & 36.0 & -0.482 & $l_{N N}=0.110 \mathrm{~nm}$ & Potoff and Siepmann (2001) \\
& $\mathrm{COM}^{\mathrm{a}}$ & - & - & 0.964 & & \\
Nanotube & $\mathrm{C}$ & 0.340 & 28.0 & - & $l_{C C}=0.141 \mathrm{~nm}$ & Jorge et al. (2002) \\
\hline
\end{tabular}

ies of quadrupolar molecules (nitrogen) adsorption on single walled carbon nanotubes. A unique slanted ordering was seen in the nanotubular systems (1-D geometry) which has not been reported before nor was present in slit nanopores (2-D geometry). It was caused by the particular combination of steric effects and the unique anisotropic attraction experienced by these fluids. However, for larger diameter SWNTs that could accommodate more than one fluid layer, differences were observed between the preferred orientations of the molecules close to the walls and those in the centre of the pores.

Thus the molecular shape is very important in modeling of adsorption in confined space with curvature because it strongly affects the structure of adsorbed phase and packing density. Thus in this study nitrogen molecule was modeled using the double LJ (but three centre) model of TraPPE type (Potoff and Siepmann 2001). Two of three centres located on nitrogen atoms of a molecule are the Lennard-Jones centres and possess point charges, while the third centre (located at a half-distance between the mentioned two) is only a point charge. The energy of interaction of a pair of molecules, with the centres separated by a distance $r$ can be written as (this energy is also a function of angular orientation):

$U(r)=\sum_{i=1}^{2} \sum_{j=1}^{2} U_{L J}^{i j}\left(r_{i j}\right)+U_{\text {electr }}(r)$

where $U_{L J}^{i j}\left(r_{i j}\right)$ is the energy of dispersion interactions between a pair of centres $i$ and $j$ (in the interacting molecules) located at the distance $r_{i j}$, and calculated from the truncated LJ potential:

$U_{L J}^{i j}\left(r_{i j}\right)= \begin{cases}4 \varepsilon_{i j}\left[\left(\frac{\sigma_{i j}}{r_{i j}}\right)^{12}-\left(\frac{\sigma_{i j}}{r_{i j}}\right)^{6}\right] & r_{i j}<r_{c u t, i j} \\ 0 & r_{i j} \geq r_{c u t, i j}\end{cases}$

where $\sigma_{i j}$ and $\varepsilon_{i j}$ are the collision diameter and the depth of the potential well for interactions between the centres of the type $i$ and $j$. The second term in (11) $\left(U_{\text {electr }}(r)\right)$ describes electrostatic interactions of a pair of molecules, described by:

$U_{\text {electr }}(r)= \begin{cases}\frac{1}{4 \pi \varepsilon_{0}} \sum_{i=1}^{3} \sum_{j=1}^{3} \frac{q_{i} q_{j}}{r_{i j}} & r<r_{\text {cut }, C} \\ 0 & r \geq r_{\text {cut }, C}\end{cases}$

where $q_{i}$ and $q_{j}$ are the values of the charges of the centres, and $\varepsilon_{0}\left(8.8543 \cdot 10^{-12} \mathrm{C}^{2} \mathrm{~J}^{-1} \mathrm{~m}^{-1}\right)$ is the dielectric permit- tivity of free space. One can observe that as previously (Furmaniak et al. 2009b), we use the cut-offs for electrostatic interactions but this is used for whole molecules (Ungerer et al. 2005) i.e. if the centres of mass of two molecules are located at a distance smaller than $r_{c u t, C}$ the sum of interactions between all pairs of charges occurring in both molecules is calculated, otherwise the electrostatic interactions are neglected. In this study this distance $\left(r_{c u t, C}\right)$ is assumed as equal to $1.5 \mathrm{~nm}$. At this distance the energy of interactions of a pair of molecules is negligibly small (the energy of electrostatic interactions for studied molecules (having quadrupole moments) decreases proportionally to the fifth power of the distance (Gray and Gubbins 1984)).

The energy of interactions between adsorbed molecules and the structure of adsorbent (each atom of a tube is treated as a LJ centre) is given by:

$U_{s f}=\sum_{i=1}^{2} \sum_{j=1}^{M C} U_{L J}^{i j}\left(r_{i j}\right)$

where $M C$ is the number of atoms forming a tube.

Table 2 collects all values of applied interaction parameters.

\section{Description of simulated isotherms using the new model}

Simulated isotherms were described using the model proposed in this study (equations (7) and (10)) and the genetic algorithm developed by Storn and Price (1996, 1997) and applied by us recently (Furmaniak et al. 2006, 2008b, 2009c). The optimized best-fit parameters are: $a_{m}, K_{1}, K_{p}$, $A_{1}, A_{p}$ and $N$, however, the tube diameter was assumed based on a histogram obtained from HRTEM results (see below). The goodness of the fit is described by the value of determination coefficient $(D C)$.

4.1 Calculation of the average number of molecules in a monolayer

For all studied systems and for the last point on the adsorption isotherm $\left(p / p_{s}=1\right)$ we repeated calculations using modified code calculating the average number of molecules in a monolayer. It was assumed that a molecule is in a monolayer if at least one of its atoms is at the distance (from any 
tube atom) not larger than $0.4 \mathrm{~nm}$ (Furmaniak et al. 2009a). The same calculations were performed for arbitrarily chosen points on the isotherms simulated for the smallest and the largest single-walled tubes. For some systems density profiles were also calculated together with histogram of angular distributions of molecules adsorbed in monolayer with respect to tube wall (we considered an angle between the axis of adsorbed molecule and the surface perpendicular to the lengthening of tube radius passing by a molecule mass centre).

4.2 The dependence between maximal monolayer capacity and tube diameter

In our previous study (Furmaniak et al. 2006) it was shown that the maximal monolayer capacity (calculated per surface unit) for nitrogen adsorption inside tubes is strongly dependent on tube diameter (especially for tubes having small diameters). For a description of this relation the equation derived from geometric considerations by Salmas and Androutsopoulos (2005) was shown to be correct. An analogous relationship describing monolayer capacity (calculated per surface unit) but for adsorption on the external surface of tubes can be easily derived for the purpose of this study:

$a_{m}^{(c)}=a_{m}^{(p)} \frac{\lambda}{2 r_{e f f} \arcsin \frac{\lambda}{2 r_{e f f}+\lambda}}$

where $a_{m}^{(c)}$ and $a_{m}^{(p)}$ are the maximal monolayer capacity on external surface of a tube having diameter $r_{\text {eff }}$ and this capacity on flat surface.

In the case of our systems we (similarly as in the case of experiment) do not express adsorption per surface area but per mass of an adsorbent. In the case of single-walled carbon nanotubes the surface density of carbon atoms in walls (calculated at the level of mass centres of carbon atoms) is the same and is equal to the surface density of carbon atoms in graphene. Bearing in mind that the bottom surface of a created monolayer is shifted by the radius of carbon atom $\left(r_{C}=0.17 \mathrm{~nm}\right)$ compared to a surface marked by centres of mass of atoms forming a nanotube, one can derive an equation analogous to (15), relating the maximal monolayer capacity (calculated per mass of adsorbent) to the effective radius of single-walled carbon nanotube:

$a_{m}^{(c)}=a_{m}^{(g)} \frac{\lambda}{2\left(r_{e f f}-r_{C}\right) \arcsin \frac{\lambda}{2 r_{e f f}+\lambda}}$

where $a_{m}^{(g)}$ denotes the maximum monolayer capacity on the surface of graphene.

\subsection{Surface areas of carbon nanotubes}

Geometric surface areas of considered nanotubes were calculated as the surface area of a cylinder having a radius equal to the effective radius of the nanotubes. Calculating surface areas from the new model (equations (7) and (10)) we assumed the specific surface area of nitrogen molecule equal to $0.162 \mathrm{~nm}^{2}$ (i.e. the value originally proposed by Emmett and Brunauer and widely accepted in literature (Rouquerol et al. 1999)).

The second approach is based on the calculation of the BET surface area. The range for the description of simulated Ar adsorption isotherms (using the BET model) was determined from the procedure proposed by Rouquerol et al. (2007). This method led to the following smallest and largest ranges of relative pressures: $0.004-0.15 p / p_{s}$ for SWNTs. A rise in the number of walls leads to the comparable ranges of the relative pressure values than mentioned above. Usually, for wider nanotubes, the operating range of pressures was shifted towards smaller values and it was almost the same.

\section{Experimental}

Three types of carbon nanotubes were studied. "Single-" and "double-walled" (see below) carbon nanotubes produced by Helix material Solution (Richardson, TX, USA) and denoted as $\mathrm{H}-1$ and $\mathrm{H}-2$, respectively, and multi-walled carbon nanotubes, Baytubes C 150 HP (Bayer Material Science, Germany) labeled as B.

Nitrogen adsorption isotherms were measured at $77 \mathrm{~K}$ using ASAP 2010 (Micromeritics, Norcross, GA, USA) sorption apparatus.

Samples were prepared for transmission electron microscopy by grinding gently and depositing on "lacey" carbon support films. The microscope employed was a JEOL 2010 , with a point resolution of $0.19 \mathrm{~nm}$, operated at $200 \mathrm{kV}$. Images were recorded photographically.

\section{The description of experimental data}

To describe experimental data of nitrogen adsorption on nanotubes we applied the same procedure as in the description of simulated data. As mentioned above, the radii of tubes were estimated from the HRTEM distributions (shown in Fig. 8). Obtained values of parameters are collected in Table 3 .

\section{Results}

\subsection{The properties of a new model}

Figure 1 shows the influence of different parameters on the plots of adsorption isotherms generated from (7) and (10). 
Table 3 The values of best-fit parameters obtained from description of experimental nitrogen adsorption data (at $77 \mathrm{~K}$ ) on commercial carbon nanotubes

\begin{tabular}{llllllll}
\hline Sample & $\begin{array}{l}r_{\text {eff }}{ }^{\mathrm{a}} \\
{[\mathrm{nm}]}\end{array}$ & $\begin{array}{l}a_{m} \\
{[\mathrm{mmol} / \mathrm{g}]}\end{array}$ & $K_{1}$ & $K_{p}$ & $A_{1}$ & $A_{p}$ & $N C$ \\
\hline $\mathrm{H}-1$ & 1.5 & 3.627 & 2018 & 0.7393 & $\sim 0$ & 0.1806 & 338 \\
$\mathrm{H}-2$ & 1.5 & 5.548 & 1783 & 0.8328 & $\sim 0$ & $\sim 0$ & 314 \\
B & 8.0 & 2.342 & 851.2 & 0.4878 & $\sim 0$ & 0.7265 & 0.9973 \\
\hline
\end{tabular}

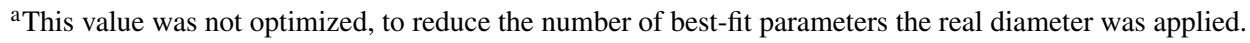
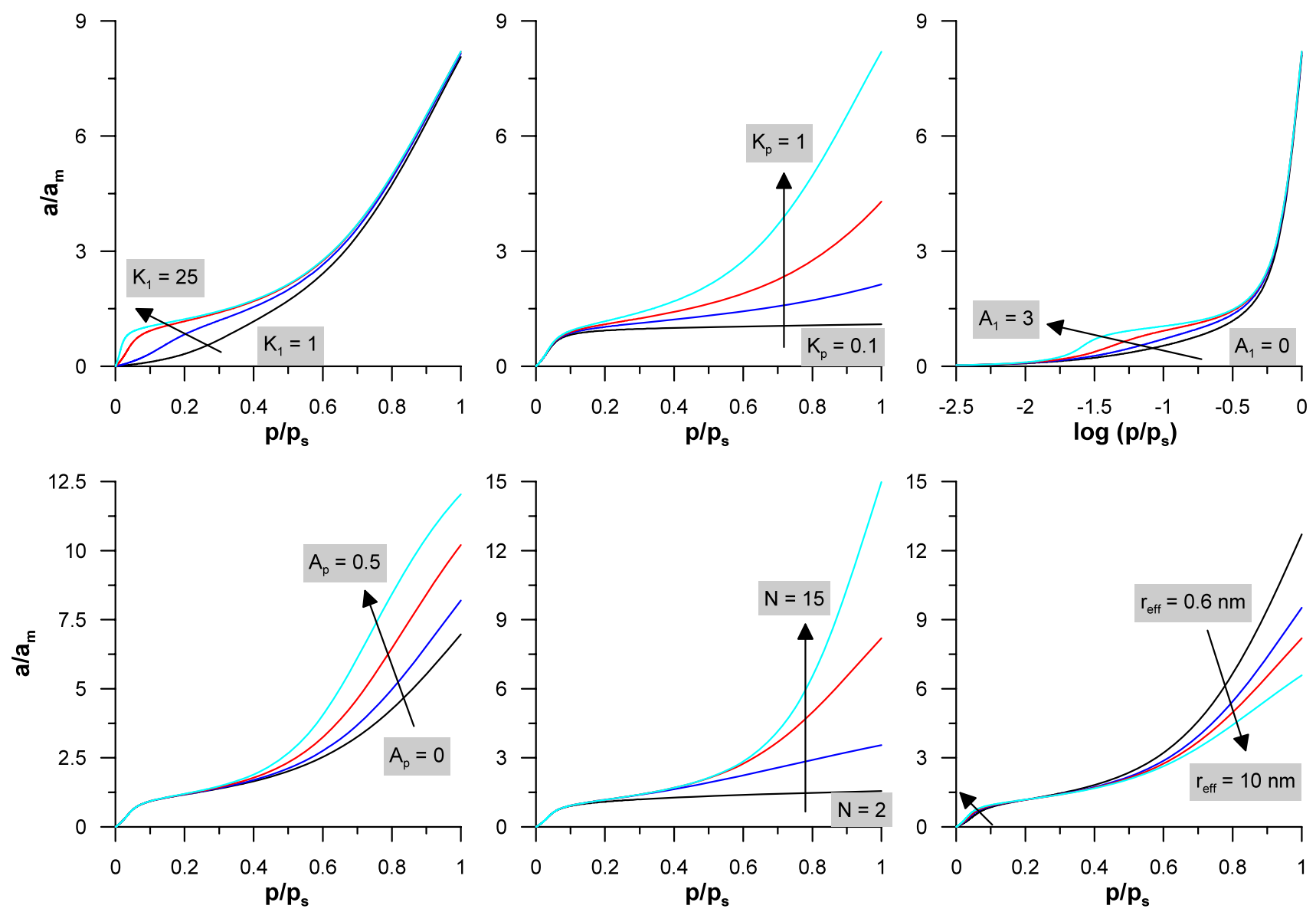

Fig. 1 The influence of parameters on the shapes of isotherms generated by new model (equations (7) and (10)); the starting parameter values are: $K_{1}=10 ; K_{p}=1 ; A_{1}=2 ; A_{p}=0.1 ; N=10 ; r_{\text {eff }}=2 \mathrm{~nm} ;$ and $\lambda=0.354 \mathrm{~nm}$

As one can observe, the rise in the value of $K_{1}$ constant changes the shape of isotherms from III up to II. This constant (i.e. Langmuir constant) is responsible for solid-fluid interactions in the first layer. On the other hand, the rise in a value of the constant $K_{p}$ (i.e. constant responsible for interaction between layers) does not influence the shape of isotherms at low pressures, but strongly increases adsorption in the multilayer region. The value of constant $A_{1}$ influences the mechanism of monolayer formation, since this constant is responsible for the fluid-fluid lateral interactions in the first layer. The rise in the value of this constant leads to a change in the shape of the isotherms (in the low-pressure range) from linear (observed for $A_{1}$ close to zero) up to $\mathrm{S}$-shaped (for large $A_{1}$ values). The influence of constant $A_{p}$ is similar as that observed for $K_{p}$. This is not surprising, since this constant is also related to the multilayer adsorption range and is responsible for the fluid-fluid lateral interactions in the second and subsequent layers. Also obvious results are observed if the influence of the number of layers is considered $(N)$, i.e. adsorption increases with 
the rise in $N$. Interesting results are obtained for the influence of the tube radius on adsorption. In the low pressure range the rise in tube size increases adsorption, however at larger pressures the opposite effect is seen. The rise in adsorption at low pressures is caused by a larger rate of desorption in the first layer (as one can see from (5) the rate of desorption for this layer is proportional to $\alpha_{1}$ value which increases with the decrease in the size of a tube-see (1)). At larger pressures this situation is also caused by the $\alpha_{i}$ values. With the decrease in tube size the values of subsequent $\alpha_{i}$ coefficients increase and this causes the rise in maximal possible adsorption in subsequent layers at the same value of $a_{m}$.

\subsection{Simulation results}

Figure S1 shows the results of GCMC simulations of nitrogen adsorption isotherms $(T=77.3 \mathrm{~K})$. One can see that for all three types of studied nanotubes adsorption at intermediate pressure values (the range of monolayer filling) decreases with the rise in tube size. On the other hand, with the rise in tube size the pressure of monolayer filling decreases. These differences are strongly pronounced for small nanotubes. Figure S2 shows the influence of the number of walls forming the tube on adsorption isotherms for an arbitrarily chosen system. One can observe that the differences vanish for the number of walls larger than three. This is why we do not consider systems containing a larger number of walls. The rise in the number of walls from 1 to 3 influences the pressure of monolayer filling as well as the monolayer capacity (however, in a small amount). In Fig. 2 we show selected snapshots (for three relative pressure values) for the smallest and for larger single walled nanotubes. One can observe the progress in creation of a monolayer (blue atoms) with the rise in relative pressure, and dependence of
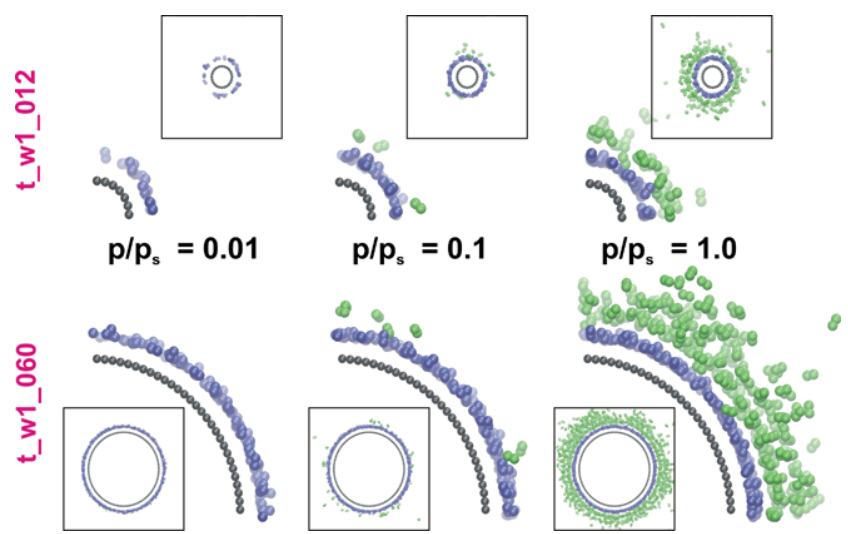

Fig. 2 (Color online) Snapshots showing the configurations of molecules adsorbed on the smallest (t_w1_012) and slightly larger (t_w1_060) single-walled carbon nanotubes (we only show central part of a simulation box, molecules adsorbed in monolayer are marked in blue) monolayer capacity as well as the number of layers on tube curvature. The latter can be also seen in Fig. S3 where we show the density profiles for the smallest and largest studied SWNT, calculated for different relative pressures. Although we do not observe major shifts in density peaks with the rise in relative pressure, Fig. $\mathrm{S} 4$ shows very interesting effect, i.e. one can observe the pressure dependence in angular orienta-
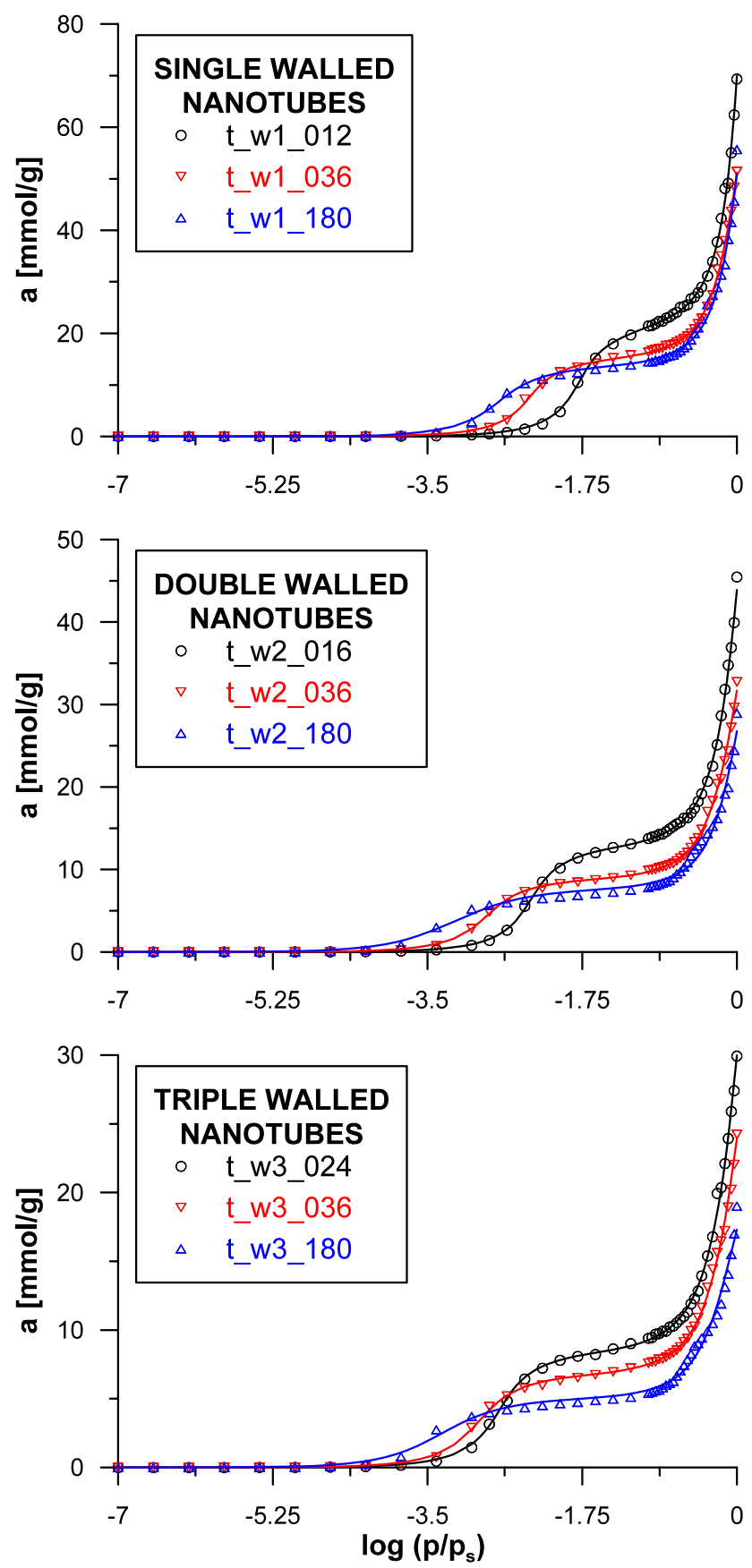

Fig. 3 Graphical representation of the fitting quality of simulated data (arbitrarily chosen systems) by the new model (equations (7) and (10)); points — simulations (only every second point is shown) and lines-model 
Fig. 4 (Color online) The plots of simulated isotherms for the smallest (t_w1_012) and the largest (t_w1_180) SWNTs, with marked adsorption in monolayer (red circles). Dashed line shows the monolayer capacity obtained from the fitting by the model proposed in this study (see Table 4)
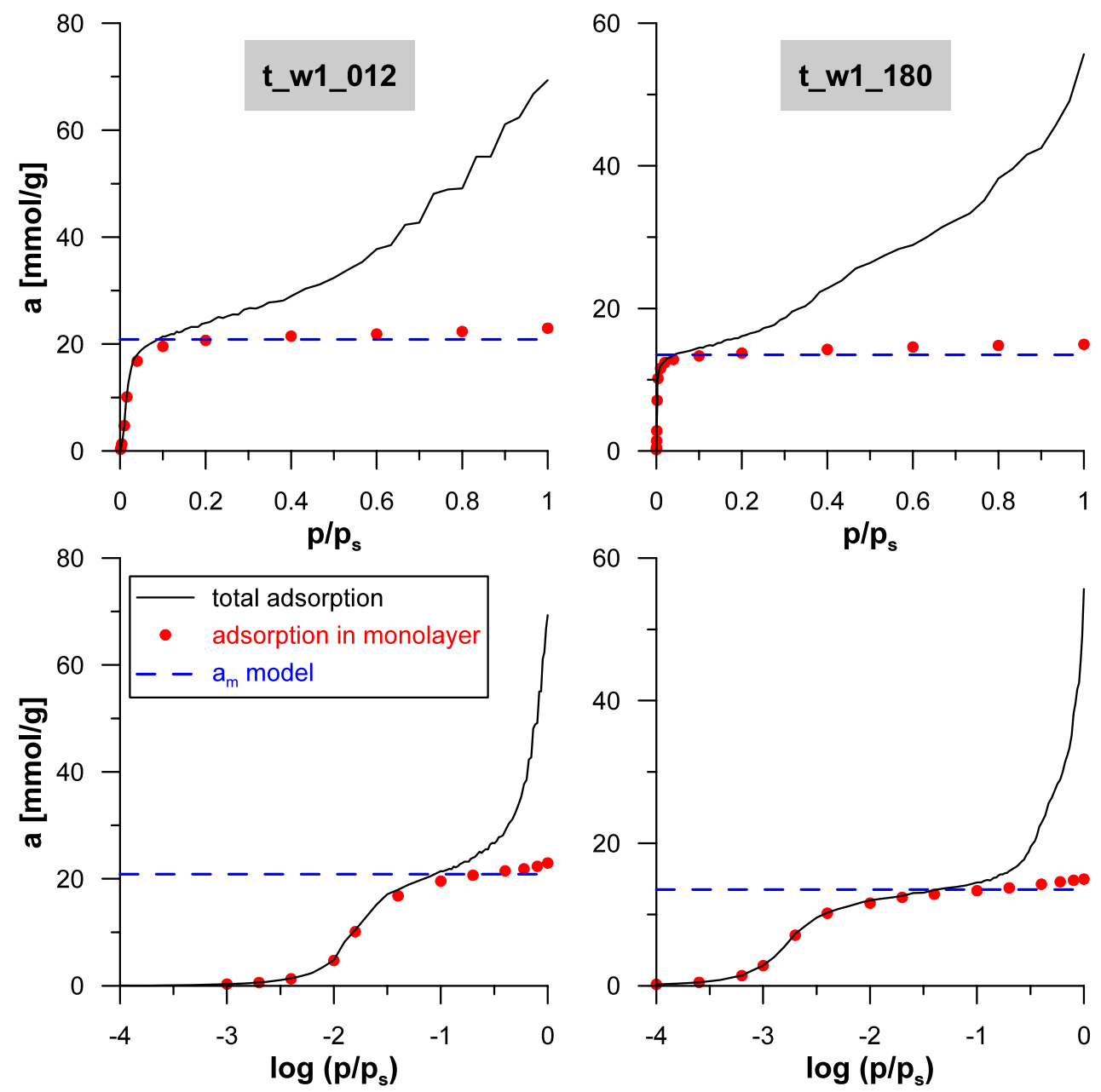

tions of some adsorbed molecules. One can see that some nitrogen molecules change the orientation in monolayer with the rise in pressure (i.e. they tend to orient parallel one to the other, and perpendicular to the tube). This is due to the minimization of the energy of adsorbate-adsorbate electrostatic interactions. Figure S5 shows that with the widening of the tube more flat orientation of molecules is preferred, and the differences between tubes are not large.

\subsection{Description of simulated data by the proposed model}

Table 3 collects the values of best fit parameters obtained from fitting of simulated isotherms using (7) and (10), and Fig. 3 shows the graphical representation for arbitrarily chosen systems. The fit is very good. Moreover, one can observe interesting correlations between different parameters, for example between $K_{1}$ and the logarithm of tube radius (see Fig. S6). This is not surprising because with the rise in tube size the surface becomes flatter and the energy of interaction of adsorbed molecule with this surface increases.
7.4 The problem of monolayer capacity

In Fig. 4 we show adsorption in a monolayer calculated using the GCMC simulation data (see above) compared with the global adsorption and monolayer capacity obtained from the application of the new model (the results for the largest and the smallest single wall nanotubes are shown). One can observe that after the preliminary monolayer filling further pressure increase leads to a small growth in monolayer adsorption due to rise in packing (caused by creation of subsequent layers) (Furmaniak et al. 2009a; Gauden 2010). Figure S7 shows the differences between simulation results and the results obtained from the new model. One can observe that simulations lead always to larger monolayer capacities compared to the model; however, the shapes of relationships (monolayer capacity vs. tube radius) are almost the same. The differences are caused by above-mentioned packing effect and, of course, by the simplifications proposed in the model.

Figure 5 shows the relations between monolayer capacity and tube radius (for single-walled carbon nanotubes) obtained from the model and from GCMC simulation data. 


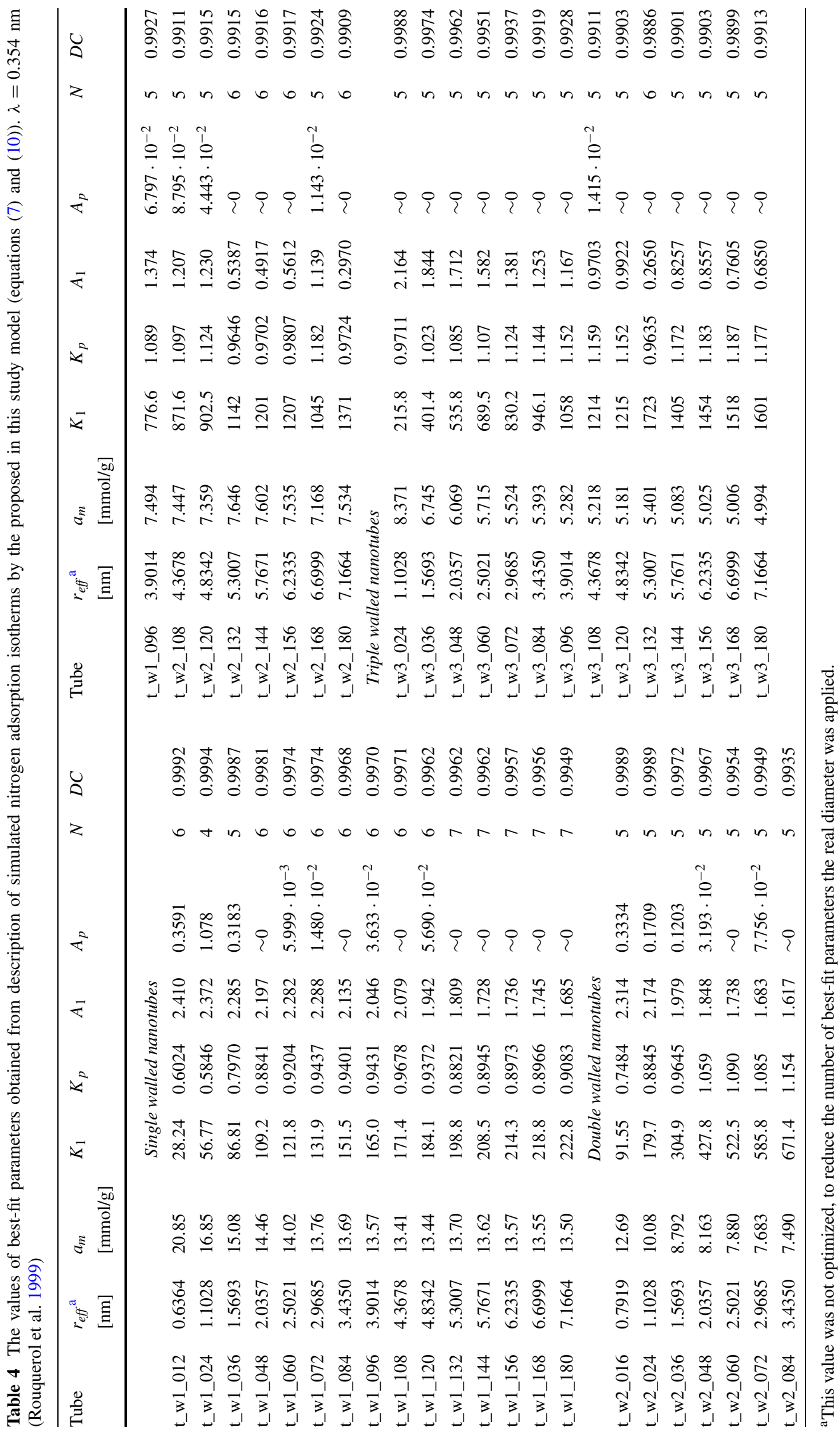


Fig. 5 The dependence between monolayer capacity and tube radius for SWNTs; left panel-monolayer capacity from the model (equations (7) and (10)); right panelmonolayer capacity estimated from simulations for $p / p_{s}=1$; solid line - theoretically predicted values form (16) (from the model-

$a_{m}^{(g)}=12.41 \mathrm{mmol} / \mathrm{g}$ and from simulation$\left.a_{m}^{(g)}=13.97 \mathrm{mmol} / \mathrm{g}\right)$

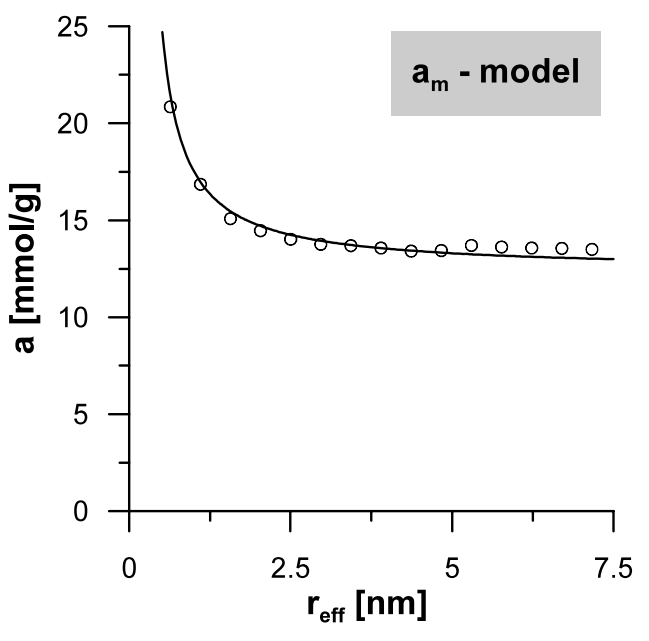

Those data were approximated by (16). The quality of fit is good; therefore the results from this figure are independent confirmation of (16) validity. As was expected, the influence of curvature on monolayer capacity is pronounced for small tube sizes. This regularity is observed not only for singlewalled carbon nanotubes but also for tubes containing larger number of walls (see Fig. 6).

\subsection{Surface areas of nanotubes}

Figure 6 shows a comparison of surface area values calculated using different methods. The values of surface areas calculated from the new adsorption equation and the BET model are similar. This is due to the shapes of obtained isotherms (of II type) with strongly marked plateau in the range of intermediate pressure values. Moreover the value of adsorption at this plateau is close to the monolayer capacity. The linearization range of the BET model applied by us partially covers the range of this plateau. On the other hand, the major advantage of our model is its capability for description of the whole isotherm.

Simultaneously, especially for larger tubes, the surface areas (calculated from the new model and using the BET) do not differ more than by few percent from the area calculated using geometric considerations. In the case of smaller tubes the situation is not so simple. As we mentioned above the monolayer capacity of small tubes (with large curvature) is much larger compared to that of a flat surface. Therefore, simply ascribing specific surface areas of molecules in monolayer leads to an overestimation of surface area. In the case of small tube it should be borne in mind that surface area can be in this case overestimated. For all cases the surface area calculated from the GCMC (and for $p / p_{s}=1$ ) is overestimated compared to geometric one. Probably the reason is improper value of nitrogen specific surface area which is pressure dependent. As we mentioned above packing effect changes the value of monolayer capacity in the whole isotherm range. A similar effect was observed for other systems (Birkett and Do 2007; Cascarini de Torre et al. 1996; Do et al. 2007; Furmaniak et al. 2009a; Gauden 2010; Wongkoblap et al. 2009).

\subsection{Description of experimental data}

Figure 7 shows representative HRTEM pictures of the studied tubes. Careful examination of the pictures leads to conclusion that the sample of so-called (by the producer) single walled nanotubes, labeled H-1 does not appear to contain single-walled tubes. It contained multiwalled tubes. The socalled double walled tube sample, labeled $\mathrm{H}-2$ does in fact contain some double-walled tubes, but mainly tubes with 3 or more layers are present. Figure 8 shows the histogram of diameters of studied nanotubes. It can be observed that the narrowest distribution occurs for $\mathrm{H}-2$ (maximum is located at $3 \mathrm{~nm}$ ). Tubes $\mathrm{H}-1$ have similar diameters to $\mathrm{H}-2$ but the distribution is wider (maximum also at $3 \mathrm{~nm}$ ). Tubes $\mathrm{B}$ are ca. five times larger (maximum at $16 \mathrm{~nm}$ ) and the distribution is the widest. As mentioned above, these maximal tube sizes were applied in the description of experimental data by the proposed model.

Before we discuss the applicability of the new model to a description of the experimental data shown in Fig. 10 we should justify the assumption of applicability of model developed to adsorption on a single tube for description of adsorption on real tube samples. It is well known that nanotubes form bundles and ropes; therefore, the process of adsorption should be dominated by so called secondary porosity created between tubes (especially if they are closed). As a consequence, as it was shown previously (Furmaniak et al. 2009a), for tubes grouped in bundles one should use the C-point method for calculation of surface area. However, careful examination of the results for bundles presented in Fig. 9 leads to interesting conclusions. In this figure we show the comparison of adsorption isotherms $\left(\mathrm{N}_{2}\right.$ at $\left.77 \mathrm{~K}\right)$ 

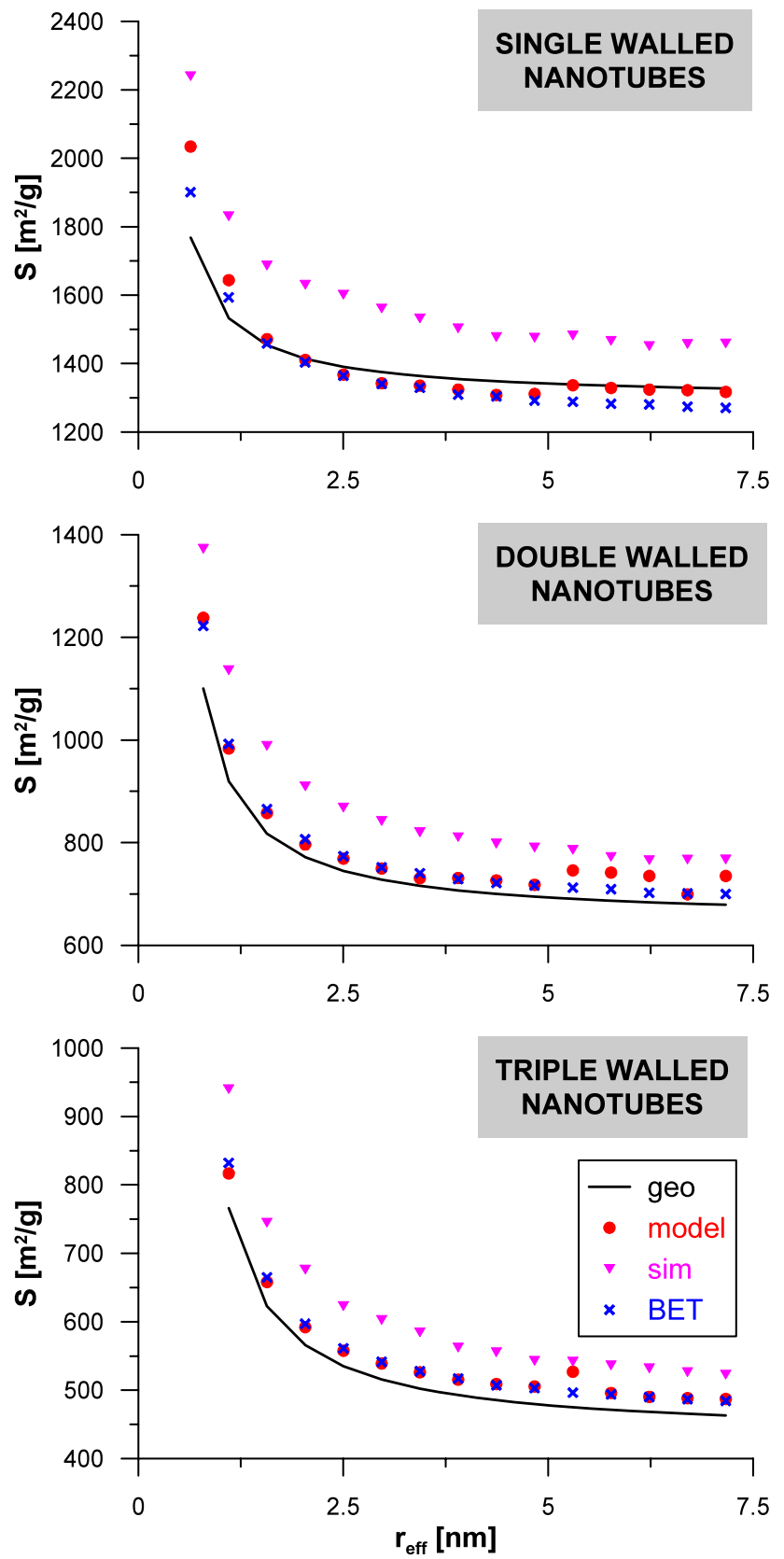

Fig. 6 The comparison of surface areas of model nanotubes with geometric surface areas (geo-geometric area, model —surface area calculated from monolayer capacity obtained by application of (7) and (10), sim - surface area calculated from simulation data basing on the number of molecules in monolayer (at $p / p_{s}=1$ ), BET-surface area calculated from BET model)

simulated for the bundle containing five tubes and for increasing separation between tubes (we used the same procedure as described previously (Furmaniak et al. 2009a)). One can observe that the shape of isotherms recorded for real samples in this study (Fig. 10) is similar to the shapes of simulated isotherms but for large distances between tubes. Moreover, on experimental data we do not observe a plateau around saturation and in fact on simulated data this plateau also vanishes on isotherms obtained for large distances between tubes. It is interesting that this plateau is usually observed on nitrogen isotherms simulated in different simulation boxes for example for square array of tubes (Yin et al. 1999). Summing up, in our opinion the experimental data obtained for the studied systems show that adsorption process can be dominated by adsorption on those fragments of bundles, where the tubes are at relatively large distances and they behave almost independently. Therefore, experimental data could be satisfactorily described by the new model proposed in this study. The results of the fitting are shown in Fig. 10 and the values of obtained best-fit parameters are collected in Table 3 . The fit is in our opinion very good. Obtained values of $A_{1}$ parameter are in all three cases practically equal to zero. This is caused by the shapes of experimental isotherms in the range of low pressures, where contrary to simulated isotherms, on experimental data we observe strongly marked Langmuirian segment. Therefore in this range the interaction of adsorbed molecules with high energy adsorption sites that are not taken into account in our model, masks the fluid-fluid interactions. The number of created layers decreases from $\mathrm{H}-1$ down to $\mathrm{B}$, moreover in the case of $\mathrm{H}-2$ the value of $A_{p}$ is also practically equal to zero.

\section{Conclusions}

Using GCMC simulations of nitrogen adsorption on single, double and triple-walled carbon nanotubes a series of adsorption isotherms were obtained. Simulated data were described using a new model proposed in this study. The model was developed from simple geometric consideration taking into account the influence of tube curvature on monolayer capacity. Since the model is very similar to the GAB approach it can be treated as nanotube analogue of the $\mathrm{GAB}$ equation however, we additionally take into account the lateral adsorbate-adsorbate interactions. The model is sufficient to describe simulated data with high accuracy moreover it leads to realistic values of monolayer capacities. Next, this new model is applied to a description of experimental data measured on a series of closed commercial carbon nanotubes. The number of best-fit parameters is reduced using the HRTEM determined tube diameter distributions. We show that for the studied carbon nanotubes the model describes experimental data very well in the whole range of relative pressures. However since at small pressures we observe Langmuirian shape on experimental data, caused probably by adsorption on impurities (amorphous carbon etc.) the model leads to negligibly small values of the parameter responsible for adsorbate-adsorbate interactions. It can be also concluded that in the case of studied three commercial nanotube samples the distance between nanotubes is 
Fig. 7 HRTEM images of studied nanotubes

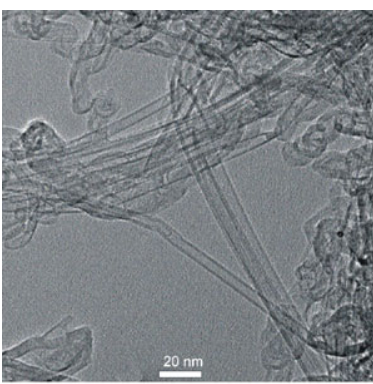

H-1

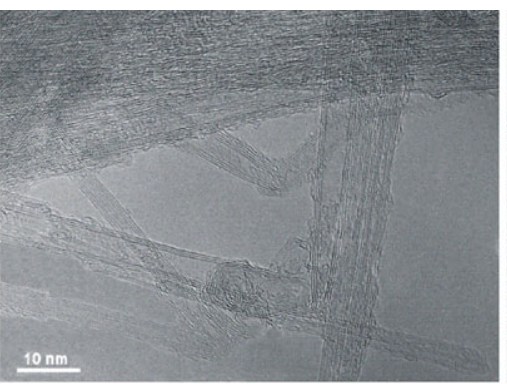

H-2

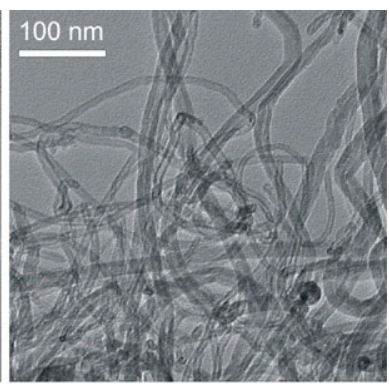

B
H-1

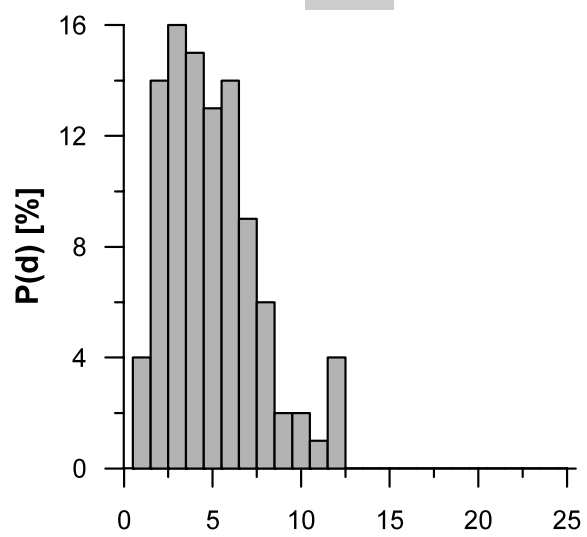

$\mathrm{H}-\mathbf{2}$

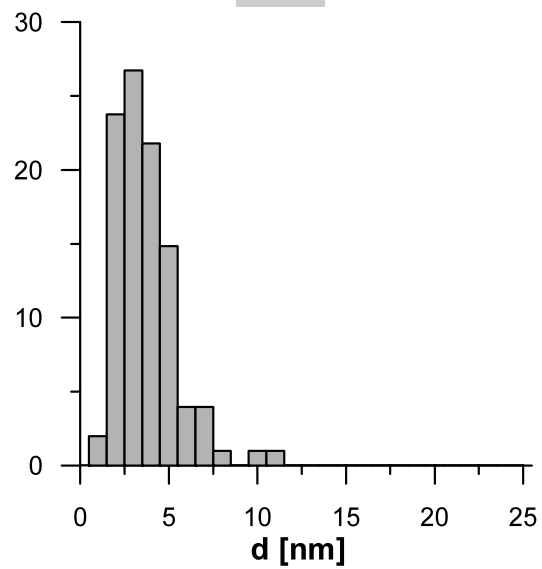

B

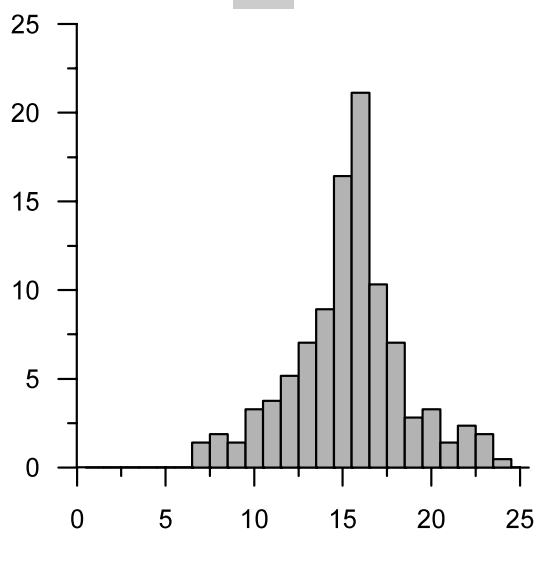

Fig. 8 Histogram of diameter distributions

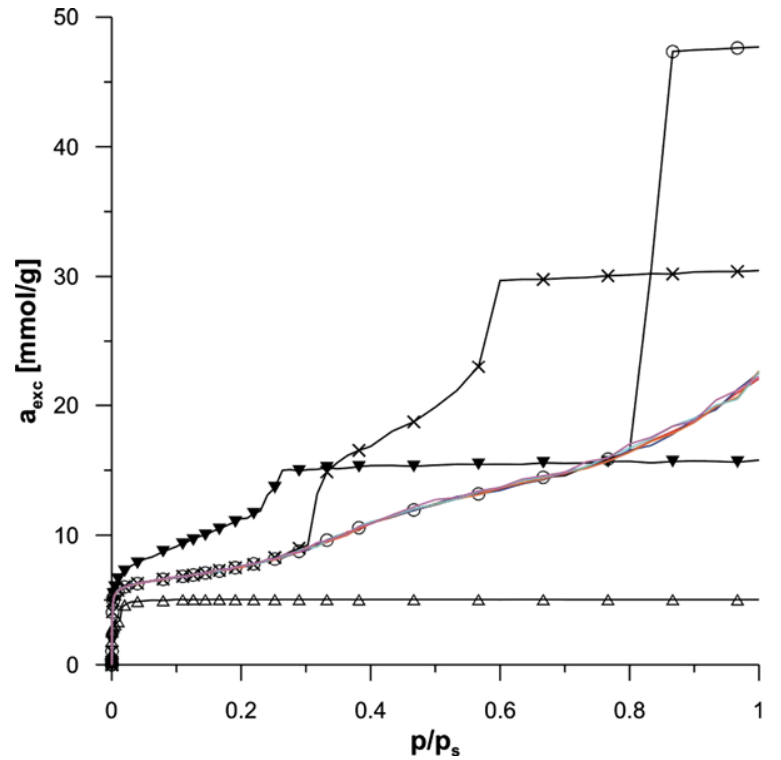

Fig. 9 The changes in shapes of nitrogen adsorption isotherms $(77.3 \mathrm{~K})$ simulated for triple-walled bundles of carbon nanotubes in the box shown above with the progressive rise in the separations between

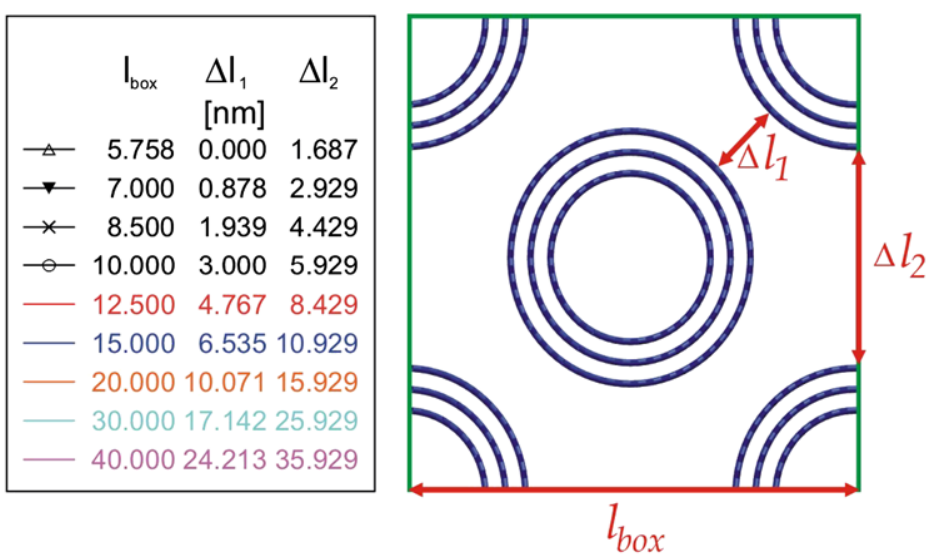

tubes (as indicated). Bundles were generated basing on triple-walled tube obtained from tubes $(48,0),(40,0)$ and $(32,0)$ - t_w3_048. Analogous boxes were applied previously (Furmaniak et al. 2008a, 2009a) 
$\mathrm{H}-1$
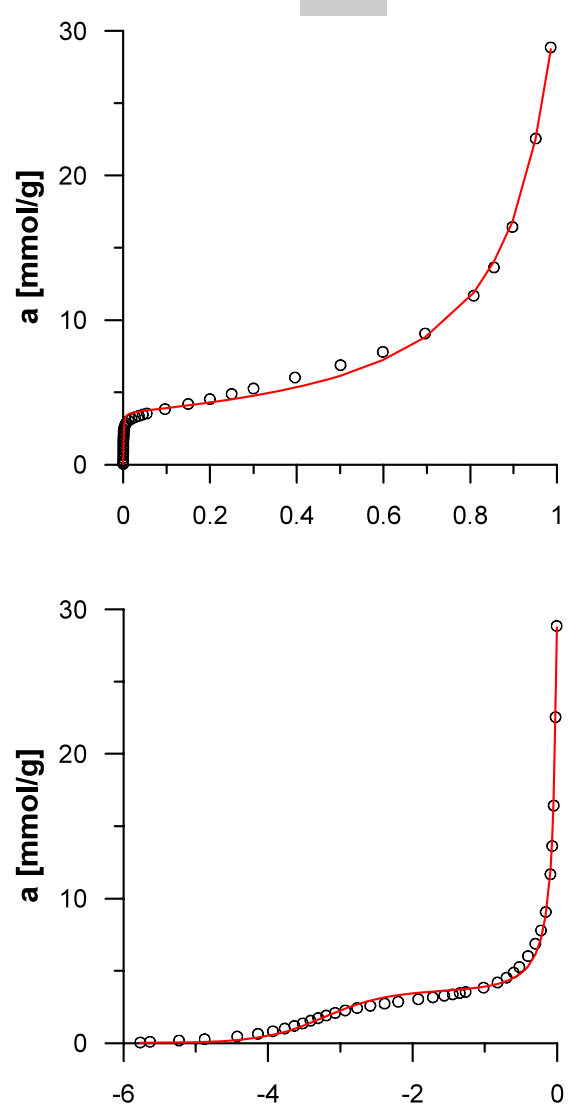

H-2

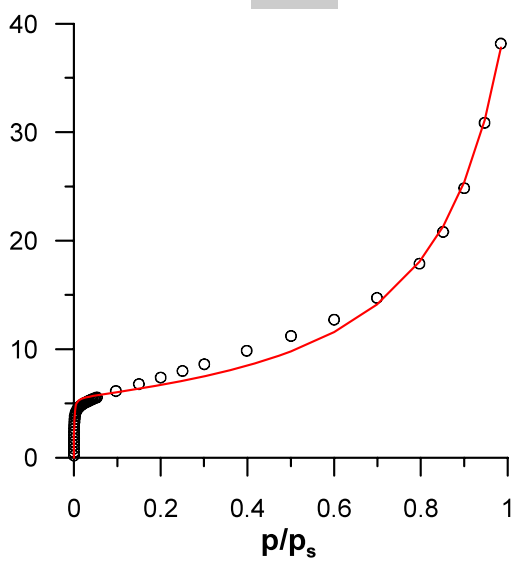

${ }^{\infty}$

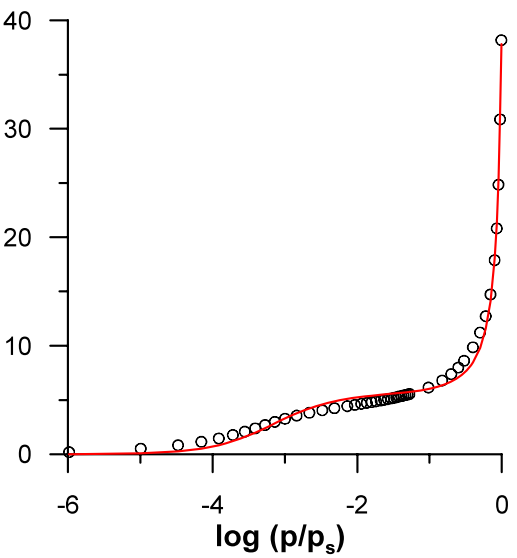

B
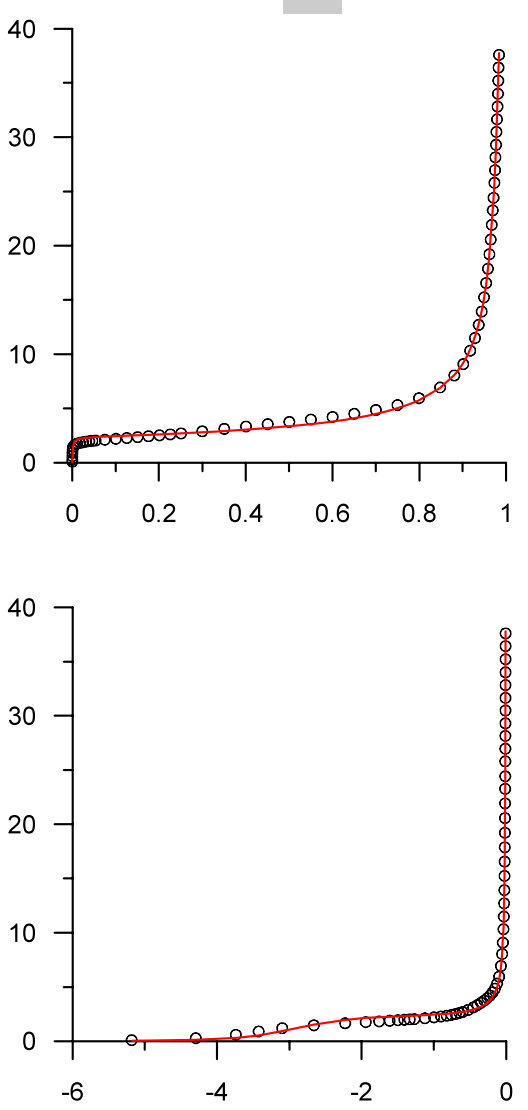

Fig. 10 The comparison of experimental data (nitrogen at $77.3 \mathrm{~K}$-circles) with obtained from fitting of the model proposed in this study (equations (7) and (10))

so large that the tubes behave almost independently, so the secondary porosity contains rather large pores, affecting the mechanism of adsorption by a small amount.

Acknowledgements The authors acknowledge the use of the computer cluster at Poznań Supercomputing and Networking Center and the Information and Communication Technology Center of the Nicolaus Copernicus University (Toruń, Poland). The project was supported by grants: N N204 009934 and N N204 288634. SF thanks the Foundation for Polish Science.

Open Access This article is distributed under the terms of the Creative Commons Attribution Noncommercial License which permits any noncommercial use, distribution, and reproduction in any medium, provided the original author(s) and source are credited.

\section{References}

Agnihotri, S., Rostam-Abadi, M., Rood, M.J.: Temporal changes in nitrogen adsorption properties of single-walled carbon nanotubes. Carbon 42, 2699-2710 (2004)

Agnihotri, S., Mota, J.P.B., Rostam-Abadi, M., Rood, M.J.: Structural characterization of single-walled carbon nanotube bundles by experiment and molecular simulation. Langmuir 21, 896-904 (2005)

Agnihotri, S., Mota, J.P.B., Rostam-Abadi, M., Rood, M.J.: Adsorption site analysis of impurity embedded single-walled carbon nanotube bundles. Carbon 44, 2376-2383 (2006)

Agnihotri, S., Zheng, Y., Mota, J.P.B., Ivanov, I., Kim, P.: Practical modeling of heterogeneous bundles of single-walled carbon nanotubes for adsorption applications: Estimating the fraction of open-ended nanotubes in samples. J. Phys. Chem. C 111, 1374713755 (2007)

Alain, E., Yin, Y.F., Mays, T.J., McEnaney, B.: Molecular simulation and measurement of adsorption in porous carbon nanotubes. In: Unger, K.K., Kreysa, G., Baselt, J.P. (eds.) COPS V. Studies in Surface Science and Catalysis, vol. 128, pp. 313-322. Elsevier, Amsterdam (2000)

Anderson, R.B.: Modifications of the Brunauer, Emmett and Teller equation. J. Am. Chem. Soc. 68, 686-691 (1946)

Arab, M., Picaud, F., Devel, M., Ramseyer, C., Girardem, C.: Molecular selectivity due to adsorption properties in nanotubes. Phys. Rev. B 69, 165401 (2004)

Arora, G., Sandler, S.I.: Air separation by single wall carbon nanotubes: Thermodynamics and adsorptive selectivity. J. Chem. Phys. 123, 044705 (2005)

Arora, G., Sandler, S.I.: Air separation by single wall carbon nanotubes: Mass transport and kinetic selectivity. J. Chem. Phys. 124, 084702 (2006) 
Arora, G., Sandler, S.I.: Nanoporous carbon membranes for separation of nitrogen and oxygen: Insight from molecular simulations. Fluid Phase Equilib. 259, 3-8 (2007a)

Arora, G., Sandler, S.I.: Molecular sieving using single wall carbon nanotubes. Nano Lett. 7, 565-569 (2007b)

Arora, G., Wagner, N.J., Sandler, S.I.: Adsorption and diffusion of molecular nitrogen in single wall carbon nanotubes. Langmuir 20, 6268-6277 (2004)

Bansal, R.Ch., Goyal, M.: Activated Carbon Adsorption. CRC Press, Boca Raton (2005)

Birkett, G.R., Do, D.D.: On the physical adsorption of gases on carbon materials from molecular simulation. Adsorption 13, 407424 (2007)

Bottani, E., Bakaev, V.: The grand canonical ensemble Monte Carlo simulation of nitrogen on graphite. Langmuir 10, 1550-1555 (1994)

Brunauer, S., Emmett, P.H., Teller, E.: Adsorption of gases in multimolecular layers. J. Am. Chem. Soc. 60, 309-319 (1938)

Cascarini de Torre, L.E., Bottani, E.J., Steele, W.A.: Amorphous carbons: Surface structure and adsorptive properties. Langmuir 12, 5399-5406 (1996)

De Boer, J.M.: The Dynamic Character of Adsorption. Clarendon, Oxford (1953)

Do, D.D., Do, H.D.: Adsorption of quadrupolar, diatomic nitrogen onto graphitized thermal carbon black and in slit-shaped carbon pores. Effects of surface mediation. Adsorp. Sci. Technol. 23, 267-287 (2005)

Do, D.D., Do, H.D., Nicholson, D.: Effects of surface structure and temperature on the surface mediation, layer concentration and molecular projection area: Adsorption of argon and nitrogen onto graphitized thermal carbon black. Adsorp. Sci. Technol. 25, 347 363 (2007)

Furmaniak, S., Terzyk, A.P., Gauden, P.A., Rychlicki, G.: Simple models of adsorption in nanotubes. J. Colloid Interface Sci. 295, 310 317 (2006)

Furmaniak, S., Terzyk, A.P., Gauden, P.A., Lota, K., Frąckowiak, E., Béguin, F., Kowalczyk, P.: Determination of the space between closed multiwalled carbon nanotubes by GCMC simulation of nitrogen adsorption. J. Colloid Interface Sci. 317, 442-448 (2008a)

Furmaniak, S., Gauden, P.A., Terzyk, A.P., Rychlicki, G.: Water adsorption on carbons-Critical review of the most popular analytical approaches. Adv. Colloid Interface Sci. 137, 82-143 (2008b)

Furmaniak, S., Terzyk, A.P., Gauden, P.A., Wesołowski, R.P., Kowalczyk, P.: Ar, C.Cl $\mathrm{Cl}_{4}$, and $\mathrm{C}_{6} \mathrm{H}_{6}$ adsorption outside and inside of the bundles of multi-walled carbon nanotubes-simulation study. Phys. Chem. Chem. Phys. 11, 4982-4995 (2009a)

Furmaniak, S., Terzyk, A.P., Gauden, P.A., Harris, P.J.F., Kowalczyk, P.: Can carbon surface oxidation shift the pore size distribution curve calculated from $\mathrm{Ar}, \mathrm{N}_{2}$ and $\mathrm{CO}_{2}$ adsorption isotherms? Simulation results for a realistic carbon model. J. Phys., Condens. Matter 21, 315005 (2009b)

Furmaniak, S., Terzyk, A.P., Gołembiewski, R., Gauden, P.A., Czepirski, L.: Searching the most optimal model of water sorption on foodstuffs in the whole range of relative humidity. Food Res. Int. 42, 1203-1214 (2009c)

Gauden, P.A.: Monolayer aspects of high-resolution $\alpha_{\mathrm{s}}$-plots. Appl. Surf. Sci. 256, 5285-5291 (2010)

Gauden, P.A., Terzyk, A.P., Furmaniak, S., Kowalczyk, P.: Adsorption potential distributions for carbons having defined pore structureGCMC simulations of the effect of heterogeneity. Adsorption 15, 99-113 (2009)

Gray, C.G., Gubbins, K.E.: Theory of Molecular Fluids, vol. 1. Clarendon, Oxford (1984)

Guggenheim, E.A.: Application of Statistical Mechanics. Clarendon, Oxford (1966)

Harris, P.J.F.: Carbon Nanotube Science. Synthesis, Properties and Applications. Cambridge University Press, Cambridge (2009)
Jiang, J., Sandler, S.I.: Nitrogen adsorption on carbon nanotube bundles: Role of the external surface. Phys. Rev. B 68, 245412 (2003)

Jiang, J., Sandler, S.I.: Nitrogen and oxygen mixture adsorption on carbon nanotube bundles from molecular simulation. Langmuir $\mathbf{2 0}$, 10910-10918 (2004)

Jorge, M., Schumacher, Ch., Seaton, N.A.: Simulation study of the effect of the chemical heterogeneity of activated carbon on water adsorption. Langmuir 18, 9296-9306 (2002)

Khan, I.A., Ayappa, K.G.: Density distributions of diatoms in carbon nanotubes: A grand canonical Monte Carlo study. J. Chem. Phys. 109, 4576-4586 (1998)

Kotsalis, E.M., Walther, J.H., Koumoutsakos, P.: Multiphase water flow inside carbon nanotubes. Int. J. Multiph. Flow 30, 995-1010 (2004)

Kowalczyk, P., Hołyst, R., Tanaka, H., Kaneko, K.: Distribution of carbon nanotube sizes from adsorption measurements and computer simulation. J. Phys. Chem. B 109, 14659-14666 (2005)

Li, F., Wang, Y., Wang, D., Wei, F.: Characterization of single-wall carbon nanotubes by $\mathrm{N}_{2}$ adsorption. Carbon 42, 2375-2383 (2004)

Lowell, S., Shields, J.E., Thomas, M.A., Thommes, M.: Characterization of Porous Solids and Powders: Surface Area, Pore Size and Density. Springer, Dordrecht (2004)

Maddox, M.W., Gubbins, K.E.: Molecular simulation of fluid adsorption in buckytubes. Langmuir 11, 3988-3996 (1995)

Marsh, T.H., Rodriguez-Reinoso, F.: Activated Carbon. Elsevier, Amsterdam (2006)

Müller, E.A.: Staggered alignment of quadrupolar molecules inside carbon nanotubes. J. Phys. Chem. B 112, 8999-9005 (2008)

Ohba, T., Kaneko, K.: Internal surface area evaluation of carbon nanotube with GCMC simulation-assisted $\mathrm{N}_{2}$ adsorption. J. Phys. Chem. B 106, 7171-7176 (2002)

Ohba, T., Matsumura, T., Hata, K., Yumura, M., Iijima, S., Kanoh, H., Kaneko, K.: Nanoscale curvature effect on ordering of $\mathrm{N}_{2}$ molecules adsorbed on single wall carbon nanotube. J. Phys. Chem. C 111, 15660-15663 (2007)

Paredes, J.I., Suárez-García, F., Villar-Rodil, S., Martínez-Alonso, A., Tascón, J.M.D., Bottani, E.J.: $\mathrm{N}_{2}$ physisorption on carbon nanotubes: Computer simulation and experimental results. J. Phys. Chem. B 107, 8905-8916 (2003)

Potoff, J.J., Siepmann, L.I.: Vapor-liquid equilibria of mixtures containing alkanes, carbon dioxide, and nitrogen. AIChE J. 47, 1676$1682(2001)$

Rouquerol, F., Rouquerol, J., Sing, K.: Adsorption by Powders and Porous Solids. Academic Press, London (1999)

Rouquerol, J., Llewellyn, P., Rouquerol, F.: Is the BET equation applicable to microporous adsorbents? In: Llewellyn, P., RodriguezReinoso, F., Rouquerol, J., Seaton, N. (eds.): COPS VII. Studies in Surface Science and Catalysis, vol. 160, pp. 49-56. Elsevier, Amsterdam (2007)

Salmas, C.E., Androutsopoulos, G.P.: Rigid sphere molecular model enables an assessment of the pore curvature effect upon realistic evaluations of surface areas of mesoporous and microporous materials. Langmuir 21, 11146-11160 (2005)

Storn, R., Price, K.: Minimizing the real functions of the ICEC'96 contest by differential evolution. In: Proceedings of 1996 IEEE International Conference on Evolutionary Computation (ICEC '96), pp. 842-844. Nagoya University (1996)

Storn, R., Price, K.: Differential evolution-a simple and efficient heuristic for global optimization over continuous spaces. J. Global Optim. 11, 341-359 (1997)

Terzyk, A.P., Gauden, P.A., Furmaniak, S., Kowalczyk, P.: Heterogeneity on high-resolution $\alpha_{\mathrm{s}}$ plots for carbon nanotubes-GCMC study. Phys. Chem. Chem. Phys. 10, 4551-4554 (2008) 
Ungerer, P., Tavitian, B., Boutin, A.: Applications of Molecular Simulation in the Oil and Gas Industry. Edition Technip, Paris (2005)

Wongkoblap, A., Do, D.D., Wang, K.: Adsorption of polar and nonpolar fluids in carbon nanotube bundles: Computer simulation and experimental studies. J. Colloid Interface Sci. 331, 65-76 (2009)

Yin, Y.F., Mays, T., McEnaney, B.: Adsorption of nitrogen in carbon nanotube arrays. Langmuir 15, 8714-8718 (1999)
Zhang, X., Wang, W., Chen, J., Shen, Z.: Characterization of a sample of single-walled carbon nanotube array by nitrogen adsorption isotherm and density functional theory. Langmuir 19, 6088-6096 (2003)

Zhang, Z., Zhang, H., Zheng, Y., Wang, L., Wang, J.: Gas separation by kinked single-walled carbon nanotubes: Molecular dynamics simulations. Phys. Rev. B 78, 035439 (2008) 